\title{
OPTIMISER LE TRAVAIL D'EQUIPE GRACE A LA MAITRISE DES LANGUES ETRANGERES / OPTIMIZING TEAMWORK BY KNOWLEDGE OF FOREIGN LANGUAGES
}

\author{
Rodica BOGDAN \\ Department of International Business, Faculty of Economic Science, University of \\ Oradea, Oradea, România \\ robogdan17@gmail.com
}

\begin{abstract}
In the context of globalization and labour mobility, it has become a habit for teams to be made up of members from different countries, speaking different languages and having an inhomogeneous cultural background. For a basic communication to achieve professional goals, a common code is usually established. However, we find that the efficiency of communication and implicitly of the work results involves the knowledge of several foreign languages. In this paper we aim to emphasise how important it is for economics students to learn different foreign languages, to assimilate and master business language. Thus, they will be able to communicate easily in a professional environment and to develop at a brisk pace in order to reach the top of the company. The ability to interact with a teammate in the language s/he has mastered since childhood leads to increased trust and better group homogeneity, which results in the optimization of teamwork.
\end{abstract}

Keywords: teamwork; foreign language; communication; labour market; mobility; globalization

\section{JEL Classification: $Y 8$}

\section{Introduction}

Afin de renforcer leur formation professionnelle à l'heure de la mondialisation, les étudiants en économie sont de plus en plus convaincus que la maîtrise d'une ou de plusieurs langues étrangères est un atout indéniable. Outre leur satisfaction personnelle d'avoir accru le capital de connaissances et de compétences diverses, ils se rendent compte qu'ils peuvent plus facilement s'intégrer dans des équipes internationales et multiethniques, dans lesquelles ils devront mettre en valeur les compétences acquises.

On parle de mondialisation depuis des décennies, et parfois le concept est trop généralisé. L'accent est mis sur les côtés économique et culturel de la mondialisation. Mais il faut aussi considérer les dimensions sociologique et anthropologique.

La mobilité de la main-d'œuvre entraîne des changements dans les comportements individuels et collectifs - la façon dont les gens interagissent dans les nouveaux contextes de travail et de proximité. Ils sont influencés même dans leurs choix vestimentaires, et leurs habitudes alimentaires, de transport et de loisirs. 
Si le premier réflexe est de penser économiquement la mondialisation, la dimension politique ne peut être sous-estimée - "La dynamique de la mondialisation est contrôlée par des forces économiques, mais ses conséquences les plus importantes sont politiques" (Klaus Müller). Les décisions sur les crises économiques, les pandémies, les déplacements de personnes dans les zones UE et hors UE, la limitation des effets de la pollution, la prévention du réchauffement climatique, etc., toutes ces décisions sont prises au niveau politique, national, international et mondial.

L'éducation de la jeune génération, la formation professionnelle, les établissements d'enseignement de chaque pays sont intégrés dans un système cohérent, socioéconomique, politique et culturel. À l'ère de la mondialisation, ce système s'ouvre indéfiniment.

Les nouveaux défis liés à la formation et à l'orientation professionnelle des membres d'une communauté diversifiée ont un impact à long terme sur toute société. Les différences culturelles, de mentalité, d'orientation religieuse et de cohésion au sein des groupes sociaux entraînent des changements dans le comportement des consommateurs et se reflètent dans la dynamique du mécanisme de l'offre et de la demande sur le marché du travail.

Les grandes entreprises cherchent à optimiser leurs coûts de main-d'œuvre, ce qui conduit dans de nombreux cas à la formation d'équipes inhomogènes, mais qui doivent fonctionner efficacement et effectuer un travail de qualité.

II ne fait aucun doute que des professionnels bien formés dans tous les domaines sont nécessaires pour répondre aux demandes dans un contexte de mondialisation économique. Leur préparation se fait par étapes, depuis l'école primaire et secondaire, jusqu'à l'enseignement supérieur et postuniversitaire, ainsi que dans la formation professionnelle, où les connaissances théoriques sont mises en pratique, sont appliquées dans des situations concrètes.

\section{Développement des compétences linguistiques}

La mission de l'enseignement supérieur est de donner à chaque étudiant la chance de devenir compétitif dans sa formation professionnelle, et même de performer. II s'agit de développer des compétences en communication orale et écrite dans une ou plusieurs langues étrangères. Nous appelons génériquement la langue d'un individu LM (langue maternelle), et pour la langue de communication dans l'environnement professionnel nous utiliserons LP. Plus les compétences linguistiques d'un professionnel sont riches (LP1, LP2, LP3, LP4, etc.), plus il sera promu rapidement et accédera aux niveaux hiérarchiques les plus élevés de l'entreprise pour laquelle il travaille.

C'est pourquoi l'éducation dans notre pays met l'accent sur l'importance de la connaissance des langues étrangères de circulation internationale et sur l'acquisition de compétences linguistiques multiples. Les étudiants ont également la possibilité d'apprendre une langue spécialisée (LS). Dans le cas des étudiants en économie, il s'agit du langage des affaires. Acquérir la capacité de s'exprimer de manière cohérente et efficace dans une langue de circulation internationale augmente les chances d'intégration dans le domaine du travail à des postes mieux 
rémunérés, dans des entreprises qui se sont développées et opèrent dans le monde entier.

\section{Langue maternelle vs. langue professionnelle}

Suite à un questionnaire sur la nécessité de maîtriser une langue étrangère pour trouver un emploi dans notre pays, nous avons reçu plusieurs réponses, dont nous allons lister deux, pertinentes pour notre étude.

Les réponses données ont mis en évidence le fait qu'au travail les gens s'adaptent pour parler une langue étrangère à des fins professionnelles (LP), mais quand il s'agit de la famille et des proches, de la communication extra-professionnelle, la plupart optent pour l'utilisation de la langue maternelle (LM). Lorsqu'il y a deux membres ou plus dans une équipe qui parlent la même LM, ils s'entraident pour rendre la communication plus efficace dans la LP.

Les questions proposées aux personnes travaillant dans des équipes multiculturelles et multilingues étaient les suivantes : 1) Dans quelle mesure est-il nécessaire de connaître une ou plusieurs langues étrangères dans le milieu professionnel ? 2) En supposant que vous utilisiez la LP au travail, si vous avez un membre de l'équipe avec qui vous parlez la même langue maternelle, préférezvous le dialogue en LM ou LP ? 3) Connaissant une ou plusieurs langues étrangères, avez-vous de meilleures chances de vous intégrer sur le marché du travail roumain?

Voici deux réponses pertinentes:

1. A.M. - Travailler pour un produit SaaS avec plus de 6 millions de clients dans le monde signifie que toute la communication doit se faire en anglais. Lorsque $Z$. a rejoint notre équipe de marketing de contenu, j'étais ravi car avoir une personne anglophone dans mon équipe serait un atout majeur pour notre activité. Le fait que tout le monde dans l'équipe de contenu, mais surtout, tout le service marketing parle couramment l'anglais, m'a vraiment aidé à intégrer Z. Pour moi, c'était un défi car nous devions basculer toute notre communication du roumain vers l'anglais, ce qui signifiait que toutes nos réunions seraient en anglais. Au début, les membres de mon équipe étaient probablement plus réticents à communiquer avec $Z$. en raison de la barrière de la langue. Pourtant, après quelques mois de collaboration, je constate que chaque membre de l'équipe est plus à l'aise de parler une autre langue au bureau. Sans oublier que la capacité de tout le monde à parler anglais s'est considérablement améliorée (Working for a SaaS product with over 6 million clients worldwide means that all the communication must be done in English. When Z. joined our content marketing team, I was thrilled because having a native English-speaking person on my team would be a major plus for our activity. The fact that everyone in the content team, but most importantly, the entire marketing department is fluent in English, really helped me integrate Z. For me, it was a challenge because we had to switch all our communication from Romanian to English, which meant that all our meetings would be in English. At first, people in my team were probably more reticent to communicate with $Z$. due to the language barrier. Still, after a few months of working together, I can see that every team member is more comfortable speaking another language at the office. Not to mention that everyone's English speaking ability improved drastically). 
2. L.T. - Je considère qu'il est hautement nécessaire de parler au moins une langue étrangère au travail, en particulier dans les domaines de l'informatique, du marketing ou des affaires, où vous pouvez avoir des collègues de différents pays avec lesquels vous devez collaborer. Pour la deuxième question, la bonne réponse serait que je mélange les deux langues. Logiquement, il est toujours plus facile d'utiliser sa langue maternelle, mais les années d'écriture et de réflexion dans la langue étrangère requises par votre entreprise laissent vraiment des traces dans votre cerveau. Parfois, même sans m'en rendre compte, je commence mes conversations avec des mots anglais, au lieu du roumain, qui est ma langue maternelle. À la troisième question, ma réponse est définitivement oui! Étant donné que de plus en plus d'investisseurs étrangers créent des entreprises ici en Roumanie, il est crucial de parler au moins une langue étrangère (anglais, allemand ou français, par exemple). Comme je l'ai déjà dit, vous devrez collaborer avec des collègues de différents pays, et nous savons tous à quel point la communication est importante. De mon point de vue, le manque de parler des langues étrangères peut vous limiter beaucoup (I consider it highly necessary to speak at least one foreign language at work, especially in the IT, marketing, or business fields, where you might have colleagues from different countries with whom you must collaborate. The correct answer would be that I'm mixing both languages. Logically speaking, it's always easier to use your mother tongue, but the years of writing and thinking in the foreign language required by your company really do leave marks on your brain. Sometimes, even without realizing, I start my conversations with English words, instead of Romanian, which is my mother tongue. Definitely, yes! Since more and more foreign investors are starting businesses here in Romania, it's crucial to speak at least one foreign language (English, German or French, for instance). Like l've said before, you'll need to collaborate with colleagues from different countries, and we all know how important communication is. From my point of view, the lack of speaking foreign languages can limit yourself very much).

\section{Optimiser le travail d'équipe}

Surtout dans les moyennes et grandes entreprises, l'optimisation du travail en équipe a pour point de départ une communication optimale entre ses membres. Une équipe multiethnique et multilingue communiquera sur la base d'un code commun (LP), un langage dans lequel les membres de l'équipe pourront s'exprimer, promouvoir leurs idées et exercer leurs compétences linguistiques.

La cohésion de l'équipe passe avant tout par le dépassement des obstacles posés par les différences culturelles, nationales ou religieuses de ses membres. Les forces et les faiblesses de chacun doivent être maintenues en équilibre, en poursuivant l'unité de but afin d'atteindre les objectifs professionnels.

En même temps, venant d'horizons différents, les membres de l'équipe peuvent apporter une touche de créativité et d'innovation en raison des différences culturelles qui les ont marqués au cours de leur éducation et de leur formation.

Toute équipe multilingue et multiethnique déclenchera des mécanismes de compétition. Ainsi, ses membres seront constamment motivés pour se surpasser et atteindre la performance.

La communication professionnelle est efficace lorsque chaque membre de l'équipe comprend ce qu'il a à faire, quelle tâche il a, et pour cela il doit très bien connaître 
le code commun, la langue parlée dans le milieu professionnel (LP). La méconnaissance des tâches fixées par le leader, résultant d'une connaissance insuffisante de la langue utilisée sur le lieu de travail peut conduire à des conflits spontanés. Les résoudre en temps opportun et continuer le travail dans de bonnes conditions nécessite l'intervention du leader, et un bon manager d'équipe doit avoir la capacité de surmonter de tels conflits. II doit poursuivre l'objectif commun à l'avenir et minimiser les lacunes des différences culturelles et la compréhension de la langue des membres de l'équipe.

\section{Conclusion}

Travailler dans une équipe multiethnique et multiculturelle est un défi pour la jeune génération à l'ère de la mondialisation. Connaître et maîtriser une ou plusieurs langues étrangères aide les étudiants en économie à s'intégrer plus facilement sur le marché du travail et à décrocher des emplois mieux rémunérés.

Dans l'enseignement supérieur de notre pays on met le point sur la nécessité de développer les compétences linguistiques des étudiants. De la part de ceux qui travaillent déjà dans des équipes internationales, le feedback est en faveur de l'apprentissage des langues étrangères et du développement des compétences linguistiques et communicatives. Ainsi, les futurs collaborateurs seront mieux préparés à accroître la cohésion d'équipe et à atteindre les objectifs de performance professionnelle. Même si la plupart des gens préfèrent parler leur langue maternelle (LM) dans un environnement non professionnel, de plus en plus de jeunes s'adaptent bien à la communication en langue étrangère (LP) dans un environnement professionnel.

\section{References}

1. Meyer, E., The Culture Map: Breaking Through the Invisible Boundaries of Global Business, Public Affairs, 2016

2. Mucchielli, R., Le travail en équipe. Clés pour une meilleure efficacité collective, ESF Editeur, 2016

3. Müller, Klaus, Globalisierung, Campus Verlag, Frankfurt/Main, 2002

4. Thomas-Bion, F., Le système éducatif français, Rue des écoles, Agora Education, Paris, 2010

5. https://www.europarl.europa.eu/news/en/headlines/economy/20190712STO5 6968/globalisation-s-impact-on-employment-and-the-eu

6. https://asana.com/fr/resources/ways-improve-team-efficiency-work

7. https://www.fond.co/blog/9-ways-multicultural-team/

8. https://www.synchroteam.fr/blog/2016/01/05/gerer-equipe-multilinguemulticulturelle/.

9. https://www.weforum.org/agenda/2018/04/fix-the-roof-now-three-prioritiesfor-the-global-economy/ 